\title{
Pulmonary Parenchyma Segmentation by Watershed Transform
}

\author{
Xing $\mathrm{Li}^{\mathrm{a}}$, Ruiping Wang ${ }^{\mathrm{a}}$, Xueqin Chen ${ }^{\mathrm{a}}$, Sha Chang ${ }^{\mathrm{a}}$ \\ ${ }^{a}$ Department of Biomedical Engineering, Beijing Jiaotong University, Beijing, China
}

\begin{abstract}
Lung cancer has become one of the leading causes of death in the world. Clear evidence shows that early discovery, early diagnosis and early treatment of lung cancer can significantly increase the chance of survival for patients. Lung Computer-Aided Diagnosis (CAD) is a potential method to accomplish a range of quantitative tasks such as early cancer and disease detection, In computer-aided diagnosis of lung disease, accurate and fast pulmonary parenchyma segmentation is the core step. Watershed algorithm is used in this paper to segment and extract lung parenchyma. To reduce over-segmentation, an improved watershed segmentation method which marks foreground and background is proposed. This method is based on watershed algorithm using "Sobel" operator on edge detection and then using mathematical morphology opening and closing operations and morphological reconstruction to mark the foreground. Extracting the local maximum associated with object will constitute the binary marker image. By testing different types of images, it proposes that the algorithm in this paper can be consistent with human visual characteristics and get more accurate, continuous object boundary. Compared with other watershed improvement methods, this proposed one requires less computational complexity, more simple parameters, and can effectively reduce oversegmentation.
\end{abstract}

Index Terms: Image segmentation, Watershed algorithm, Mathematical morphology, Sobel operator

(C) 2011 Published by MECS Publisher. Selection and/or peer review under responsibility of the Research Association of Modern Education and Computer Science.

\section{Introduction}

In order to improve the survival rate of lung cancer patients, early detection has a significantly more hopeful prognosis and is the key treatment. CT scanning presents great opportunities for lung cancer diagnosis. However, the large amount of CT images caused increasing work and inevitable false diagnosis rate. Image processing techniques, for example segmentation and extraction of nodules, reconstruction and rendering of suspicious objects, make it possible that computer-aided reading images, help doctors to analyze pathological changes and other regions of interest in character and even in accurate quantity, and release the doctors' burden.

* Corresponding author.

E-mail address: 
Pulmonary parenchyma segmentation is the first step of a computer-aided diagnosis system for early detection of lung cancer. Several automatic and semi-automatic lung segmentation methods have been proposed in the literature. Rikxoort et al. [1] and Zhang et al. [2] proposed an automatic lobe segmentation method based on an anatomic pulmonary atlas. Rikxoort et al. [3] introduced another automatic lobe segmentation approach including a supervised fissure enhancement filter and grouping of adjacent fissure voxels into plates. Ross et al. [4] proposed a semi-automatic lobe segmentation in which fissures are calculated by a thin plate spline method based on markers set interactively on the fissure. Wang et al. [5] applied semi-automatic 2D fissure enhancement and 3D fissure interpolation to segment the lung into lobes.

This paper also focuses on computer-aided segmentation of pulmonary parenchyma. The watershed transform is used to segment the lung. Since the separation of the left and right lungs and extraction of the borders are automatically done by the technique, the computation time is highly reduced.

\section{Methods}

Watershed transformation is a common technique for image segmentation. It is a classical and effective segmentation method by which one-pixel-wide continuous edge can be extracted. More importantly, it has the advantages of high segmentation precision and accurate positioning. Its drawbacks include over-segmentation and sensitivity to noise [6]. Currently, there are two main methods to solve the problems. The first one is postprocessing for the segmented images. But this method is time-consuming and its computation is much large. The second one is preprocessing, belonging to the watershed segmentation algorithm based on the marker. According to V. Grau et al[7], marker-based watershed algorithm is more effective to solve the oversegmentation. Therefore, Marker-based Watershed Transform is tried in this paper.

\subsection{The principle of Watershed Transform}

Generally, the watershed transform is computed on the gradient image, where the boundaries of the catchment basins are located at high gradient points. The principle of the immersion-based watershed algorithm can be illustrated by imaging the gradient image as a relief, with the 'height' variable being the grey-value for each pixel position. Imaging, water immersing from the bottom of the relief (the darkest parts), every time the water reaches a minimum which corresponds to a region in the original image, a catchment basin is 'grown'. When two neighboring catchment basins eventually meet, a dam is created to avoid the water merging at that level of flooding and spilling from one basin into the others. We utilized this property to make the left and right lung separate during the transformation. When the water reaches the maximum gray-value, the union of all dams forms the watershed lines.

\subsection{Marker-based Watershed Transform}

Over-segmentation is a well-known drawback in watershed segmentation. This phenomenon is more serious by applying standard watershed transform on the gradient image of a pulmonary CT slice (fig.(b)).

By using marker-based watershed transform proposed in this paper, we can decrease the regional minima and bound them within the regions of interest to prevent over-segmentation.

The details of our approach are described in the following steps.

- First, read an image and the original one is transformed into a gray image (fig.(a)).

- Watershed transform is applied on the gradient image, so the step is to obtain the gradient image (right one of fig.(a)). The Sobel masking operator is applied on the CT gray image in both horizontal and vertical directions to create the gradient image. Watershed algorithm can be directly tested on it, and then its segmentation result will be as a contrast (fig.(b)). 
- If the over-segmentation is serious, the foreground and background should be marked. Select a circular structure element and then apply open morphological operation and morphological reconstruction (fig.(c)).

- Following, apply morphological clearance operation and reconstruction to obtain its complement image (fig.(d)).

- A local maximum (display the maximum areas in original image) (fig.(e) and fig.(f)).

- Again apply an appropriate open and clearance operator to modify the local maximum areas (fig.(g)).

- The modified image then is changed into a binary image (fig.(h)).

- Finally, apply watershed transform on the binary image.

\section{Result and Discussion}

Repeated experiments show that, different structure elements will contribute to different image processing results. By testing many structure elements, the most appropriate one has been selected. Fig.show some results tested on a $512 \times 512 \mathrm{CT}$ image which mainly includes solitude lung nodules.

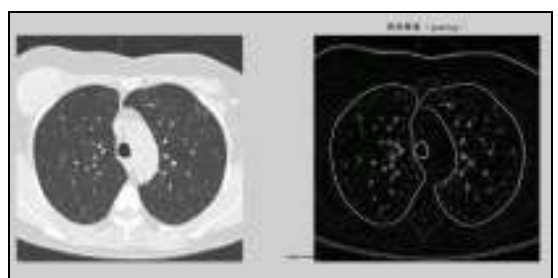

(a)

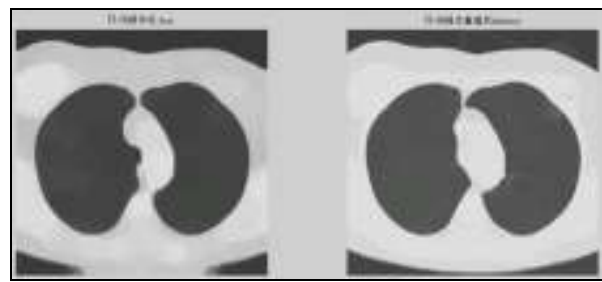

(d)

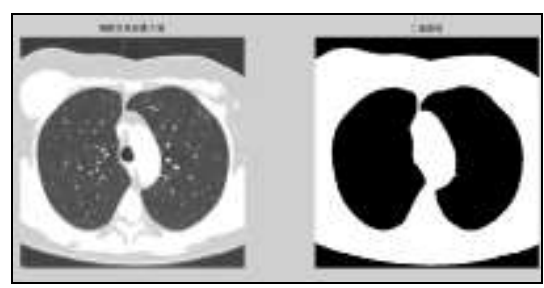

(g)

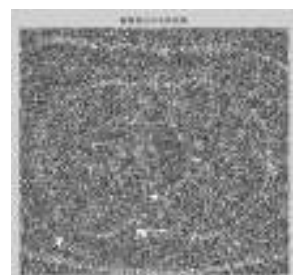

(b)

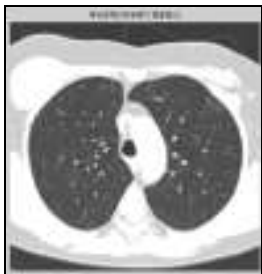

(e)

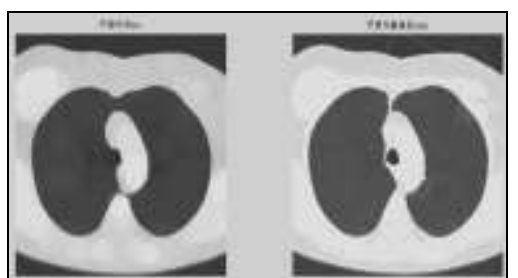

(c)

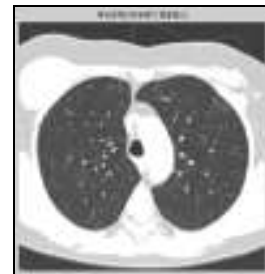

(f)

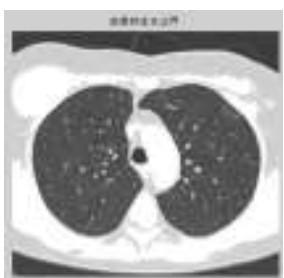

(h)

Fig. (a) The gray image and gradient image. (b)Apply watershed algorithm directly without any preprocessing. The picture shows the serious over-segmentation. (c)Open and reconstruction operations. The two pictures show that, its outline is clearer after reconstruction. (d)Morphological clearance operation and reconstruction based on the picture c. The outline is kept complete, and remove some disrelated objects effectively. (e)Local maximum. (f)Local maximum displayed on the original image. (g)The modified local maximum and binary image. (h)After watershed transform. 
In order to evaluate the performance of proposed method, six benign and three malign cases containing 230 CT slices are collected. They are the same thickness with 5 millimeters from the National Cancer Imaging Archive that were collected by the Lung Image DataBase Consortium (LIDC).

From the above results, it can be seen that morphological operations can effectively improve the edge information, make outlines more clear and the original image integrate. Besides, the segmentation result is better in reducing over-segmentation.

In the realization of segmentation, several other methods have been also tried, such as iterative threshold and OTSU method. However, because of its vast time-consuming, iterative threshold does not apply to realtime processing; OTSU method which is based on the bimodal histogram, is practical only when objective and background intensity have a distinct difference. By comparing these segmentation results of the methods and their time-consuming (Table 1), marker-based watershed transformation is the most effective.

Table.1. Time-consuming comparison of three methods

\begin{tabular}{|c|c|}
\hline Method & $\begin{array}{c}\text { Average processing time } \\
\mathbf{( 5 1 2} \times \mathbf{5 1 2} \text { x16) }\end{array}$ \\
\hline Iterative threshold & Nearly $17 \mathrm{~s}-65 \mathrm{~s}$ \\
\hline OSTU & Almost $8 \mathrm{~s}-25 \mathrm{~s}$ \\
\hline $\begin{array}{c}\text { Marker-based } \\
\text { watershed transform }\end{array}$ & nearly $4 \mathrm{~s}-12 \mathrm{~s}$ \\
\hline
\end{tabular}

\section{Conclusions}

The recent studies demonstrate that the lung segmentation method as a preprocessing step for CAD system may affect CAD results. An automated lung segmentation method using watershed transform has been developed in this paper, which is accurate, fast and can be parallelized to make it less time-consuming for the high volume datasets.

Even if there are no strong edges between the markers, our technique always detects a significant edge in the desired area that demonstrates the robustness of this technique.

The above experimental results show that different structural elements can contribute to different results in extracting edge and reserving information. To the lung CT images elected in the experiment, radius of 20 round structure element is ideal.

According to repeated testing on different types of images, this segmentation algorithm can obtain an accurate and continuous object boundary, which conforms to human visual features, and practical significance. Besides, this algorithm has low requirements for computational complexity, simple parameters, and can effectively reduce over-segmentation.

\section{References}

[1] E.M. van Rikxoort, M. Prokop, B. de Hoop, M.A. Viergever, J.P.W. Pluim, and B. van Ginneken, "Automatic segmentation of the pulmonary lobes from fissures, airways, and lung borders: evaluation of robustness against missing data," in MICCAI, 2009, pp. 263-271.

[2] L. Zhang, E. A. Hoffman, and J. M. Reinhardt, "Atlasdriven lung lobe segmentation in volumetric X-ray CT images," IEEE Trans Med Imaging, vol. 25, no. 1, pp.1-16, 2006.

[3] E. M. van Rikxoort, B. de Hoop, S. van de Vorst, M. Prokop, and B. van Ginneken, "Automatic segmentation of pulmonary segments from volumetric chest CT scans.," IEEE Trans Med Imaging, vol. 28, no. 4, pp. 621-630, 2009.

[4] J. C. Ross, R. San Jose Estepar, A. D'iaz, C.-F. Westin, R. Kikinis, E. K. Silverman, and G. R. Washko, 
"Lung extraction, lobe segmentation and hierarchical region assessment for quantitative analysis on high resolution computed tomography images," in MICCAI, 2009, pp.690-698.

[5] J. Wang, M. Betke, and J. P. Ko, "Pulmonary fissure segmentation on CT," Medical Image Analysis, vol. 10 , no. 4, pp. $530-547,2006$.

[6] H.P.Ng, S.H.ong, K.W.C.Foong, P.S.Goh, and W.L.Nowinski, "Medical image segmentation using Kmeans clustering and improver watershed algorithm," in Proc. IEEE Southwest Symposium on Image Analysis and Interpretation, pp.61-65, 2006.

[7] V. Grau, A. U. J. Mewes, M. Alcañiz, R. Kikinis and S. K. Warfield, "Improved Watershed Transform for Medical Image Segmentation Using Prior Information”, IEEE Transactions on Medical Imaging, Vol. 23, No. 4, April 2004

[8] W. A. Kalender, H. Fichte, W. Bautz and M. Skalej, "Semiautomatic evaluation procedures for quantitative CT of the lung." J Comput Assist Tomogr Vol.: 15 pp: 248-255, 1991.

[9]Computer assisted Diagnosis for Lung Cancer Screening: http://www.cvio.ioiiisvilie.edu.

[10] J. B. T. M. Roerdink and A. Meijster, "The watershed transform: Definitions, algorithms and parallelization strategies," Fundamenta Informaticae, vol. 41, pp. 187-228, 2001.

[11] A. N. Moga and M. Gabbouj, "Parallel marker-based image segmentation with watershed transformation", Journal of Parallel and Distributed Computing 51, 27\{45\}, 1, 1998

[12] E. A. Hoffman and G. McLennan, "Assessment of the pulmonary structure-function relationship and clinical outcomes measures: Quantitative volumetric CT of the lung," Academic Radiol., vol. 4, no. 11, pp. 758-776, 1997.

[13] L. W. Hedlund, R. F. Anderson, P. L. Goulding, J. W. Beck, E. L. Effmann, and C. E. Putman, "Two methods for isolating the lung area of a CT scan for density information," Radiology, vol. 144, pp. 353357,1982 .

[14] S. G. Armato III and W. F. Sensakovic, "Automated Lung Segmentation for Thoracic CT: Impact on Computer-Aided Diagnosis”Academic Radiology: Vol.11, No. 9, pp. 1011-1021, September 2004.

[15]M. S. Brown, M. F. Mcnittgray, N. J. Mankovich, J. G. Goldin, J. Hiller, L. S. Wilson and D.R. Aberle, "Method for segmenting chest CT image data using an anatomical model - preliminary results", IEEE Trans Med Imaging. Vol. 16, pp. 828-839, Dec.1997.

[16] L. Vincent and P. Soille, "Watersheds in digital spaces: An efficient algorithm based on immersion simulations." IEEE Trans.Patt.Anal.Mach.Int. 13(6), pp. 583-598, June 1991.

[17] K. Haris, S. N. Efstratiadis, N. Maglaveras, and A. K. Katsaggelos, "Hybrid image segmentation using watersheds and fast region merging," IEEE Trans. Image Processing, vol. 7, No. 12, pp.1684-1699, Dec. 1998. 